\title{
Patient recruitment for clinical trials on traditional Chinese medicine: Challenges, barriers, and strategies
}

\author{
Cheng King-Fai \\ Leung Ping-Chung \\ Wong Lai-Yi \\ Fong Yuet-Shim
}

Centre for Clinical Trials on Chinese Medicine, Institute of Chinese Medicine, The Chinese University of Hong Kong, Hong Kong, China
Correspondence: Leung Ping-Chung Director of Institute of Chinese Medicine, The Chinese University of Hong Kong, 5/F School of Public Health Building, Prince of Wales Hospital, Shatin, Hong Kong, China

Tel +85222528868

Fax +852 2632544 I

Email pingcleung@cuhk.edu.hk
Background and objective: Failure to recruit sufficient numbers of participants is a major barrier to the completion of randomized controlled trials in traditional Chinese medicine (TCM) clinical trials. The purpose of this paper is to analyze the commonly used strategies for the recruitment of patients in TCM clinical trials, to identify the most common reasons for attrition and refusal, and to identify barriers and potential solutions to the difficulties.

Methods and results: There are four stages in the actual recruitment process, which included introducing the project to the potential patients, explaining how to implement the project, assessing and intensifying the understanding and facilitating patient decision-making. When insufficient recruitment occurred, the following steps should be considered: reevaluating the required sample size; adding new sites to the trial; eliminating hospitals that had poor recruiting records; extending the patient recruitment period, modifying the patient inclusion/exclusion criteria; and shifting placebo-controlled to active-controlled arrangements. Success in reaching target recruitment depended largely on being able to directly contact patients through posters, newspaper advertisements, television interviews, patient support groups, and physician referrals in hospitals.

Conclusions: Suspicions against the placebo and unwillingness to stop taking other herbal supplements made recruitment more difficult, time-consuming, and costly. In a Chinese community, open advertising in the local newspaper may be particularly attractive.

Keywords: recruitment, randomized controlled trials (RCT), traditional Chinese medicine (TCM)

\section{Introduction}

The randomized controlled trial (RCT) is widely accepted as the most powerful research method in the evaluation of the efficacy of a study medication (Ross et al 1999). These principles are also applied to traditional Chinese medicine (TCM) studies (Leung et al 2008). Efficient and effective recruitment of patients and retention of participants are essential steps in the clinical trials. However, it has been shown that the most eligible patients do not participate in clinical trials. A study reported that in eligible patients who were aware of an appropriate clinical trial, 71\% choose not to participate (Anderson 2001). In some RCTs, the dropout rate was as high as 80\% (Kemmler et al 2005). The results of recruitment are generally influenced by a number of factors, including the nature of the study itself, the recruitment strategy used, and the target population (Taylor-Davis et al 1998). In this paper, we examine some particular barriers to patient participation and explore possible solutions.

TCM and herbal medications have remained very popular in Chinese societies around the world because it has developed within a culture that has endured for more than 3000 years over a vast geographical area with an enormous population. Centuries 
of empirical studies and accumulated experience has retained good and useful medical practices while what was not effective was discarded. There still remains an high incidence of side effect and toxicity as well as TCM not providing expected benefits. Nowadays, when modern medicine fails to provide the expected benefits, TCM could be considered as a last resort; so an unrealistic perspective often exists in participants. It may therefore be easier to conduct TCM clinical trials in the Chinese community; however, barriers or difficulties still exist.

\section{Process of recruitment}

It is important to define clearly the target population to be studied, which depends on the study protocol. The difficulty of recruitment depends on the exclusion/inclusion criteria, age, study medication, disease risk factor, biomarkers tested, stage of disease, etc. In our TCM clinical trials, the target populations are usually those with chronic diseases or with their conditions under control. Modern medicine has failed to obtain perfect solutions for chronic diseases or conditions that include allergic conditions, autoimmune diseases, cancers, chronic pain, chronic derangements, degenerative diseases, nerve damages, viral infections, etc. Our experiences tell us these patients would be willing to participate in TCM clinical trials because modern medicine is unable to help them.

There are four stages in the actual recruitment process, which are clearly dynamic and interactive (Harris and Dyson 2001). These stages include:

1. Introducing the project to the potential patients

2. Explaining how to implement the project

3. Assessing and intensifying the understanding

4. Facilitating patients' decision-making.

The process of screening started at the time of initial contact with the study team, either in person or over the telephone. The initial approach is considered vital. It is essential that someone is assigned to telephone duty to deal with initial contact to avoid loss of potential volunteers. The primary purpose is to put the patient at ease and to establish rapport between patient and research team quickly. Table 1 lists our means that we usually use in our clinical trial center during the initial approach stage.

When subjects called in response to the initial approach, the research nurse noted down their names, a contact telephone number to start screening on related eligibility criteria, and whether they were using an herbal preparation. If a candidate who was using any herbal preparation was willing to participate the trial, our research nurse would advise the candidate to stop the herbal preparation for a suitable period (washout period) according to the requirement of the study protocol before they are enrolled. If the telephone screening criteria were met, the research nurse would arrange a proper interview for informed consent. Participants then progressed to laboratory testing for necessary function assessments. If the inclusion/exclusion criteria were satisfied and informed consent forms were obtained, the participants progressed to randomization to either TCM treatment group or placebo group.

\section{Barriers and strategies of recruitment}

Explicit inclusion/exclusion criteria are essential elements of any clinical studies. According to the trial protocol, potential subjects for TCM trial had to satisfy the inclusion/exclusion criteria. Their participation was quite different from visiting their general practitioner. If the inclusion/exclusion criteria of TCM clinical trials were too rigorous, the difficulty of recruitment is increased. For example, we had conducted a RCT by using a Chinese herbal formula named DBT to

Table I Initial approach to patients

\begin{tabular}{lc}
\hline Initial approach & Our experience \\
\hline Advertisement (newspapers) & TCM for postmenopausal symptoms \\
Public seminars & Herbal formula for lung and colon cancer \\
Published articles (usually in newspapers) & TCM for postmenopausal symptoms \\
Television interview & TCM and postmenopausal symptoms \\
Physician referral & TCM for diabetic foot ulcer \\
Outpatient/inhouse physician referral & TCM for influenza prevention \\
Friend or acquaintance referral & TCM for influenza prevention \\
Nonprofit and nongovernment organizations (NGO) & Herbal formula for lung and colon cancer \\
Community (letters and posters) & Acupuncture for smoking cessation, osteoporosis study \\
University collaboration & TCM formulas for post-stroke and insomnia
\end{tabular}

Abbreviation: TCM, traditional Chinese medicine. 
control post-menopausal symptoms. The protocol required that subjects should have at least 14 hot flushes per week and had not undergone any treatment including hormone therapy and herbal treatment, before they entered the study and during the study period. We initially approached 452 candidates, after screening, only 60 subjects were eligible, finally only 52 subjects completed the clinical trial (Table 2). Sometimes, we needed to identify particular Chinese herbs which were consumed by participants themselves to judge if there were any interaction with the study TCM medication. In this case, we advised the subjects to stop using these herbs before they were enrolled and during study period. However, many candidates were unwilling to do so. To deal with this problem, we advised the candidate keep using the herbal preparation during study period, if our TCM practitioner found that the herbal preparation would not interfere with study herbal medication. To minimize the outcome bias, we treated this case very carefully. If we could not ensure whether the herbal preparation taken by the candidate had any interaction with the study herbal medication, and the candidate was unwilling to stop taking his/her herbal preparation, we had to exclude the candidate.

The recruitment procedures are not straightforward. To enhance recruitment through television interviews, newspaper releases, and advertisements to make use of media would be very useful. However, to arrange screening interviews with patients and retaining them in the trial without drop out was even more difficult.

Liaising with local organizations and collaborating with local practitioners would be helpful for recruitment, especially when nonprofit organizations were involved. Referral from physicians was another channel of recruitment, but it had not been a main channel in Hong Kong. In our experience, recruiting patients through the health services (hospital consultants, general practitioners, and clinics) is likely to be less successful because professional heath specialists had other priorities.

In general, the means of initial contact with potential volunteers that we targeted in our TCM clinical trials

Table 2 Patient recruitment for a traditional Chinese medicine formula trial

\begin{tabular}{ll}
\hline Process of recruitment & Case \\
\hline Initial approach & 452 \\
Assessed for eligibility & 71 \\
Screen failures & $1 \mathrm{I}$ \\
Enrolled/randomized & 60 \\
Drop out & 9 \\
Completed & 52 \\
\hline
\end{tabular}

included advertisements and relevant articles in newspapers, public seminars, television interviews, announcements in community centers, etc (Table 1). Appeals in newsletters may also be helpful but not as the primary channel for recruitment. According to our experience, advertising in local newspapers and announcements in community centers are effective. But an optimal approach should depend on the nature of the study. For example, announcements in community centers were efficient for nonspecific diseases/conditions such as a smoking cessation trial and menopausal symptoms. Considering the main channel of initial approach should be depend on the nature of study disease/condition. For the diseases/symptoms eg, postmenopausal symptoms, that the public have been aware of and with a higher prevalence, the initial approach through advertisement would be more efficient. For those that were specific or rare in prevalence, eg, diabetic foot ulcer, a referral by physicians was the best way. For most diseases/conditions, multiple approaches were needed.

Poor clinical trial recruitment and retention impede the successful evaluation of new and existing interventions, and delay processes of clinical implementation (Spilker and Cramer 1992). Patients may not want to take a chance with randomization in an environment where the patients' enthusiasm is often in favor of TCM and against receiving a control treatment or no treatment. Another cause of low recruitment especially for TCM trial is the dosage form and taste of the study herbal preparations. Dosage forms of TCM usually include capsules, tablets, pills, sachets, and oral liquids. If the study drug was prepared in oral liquid and the taste was not good, and a large amount of doses was required to consume, the difficulty of recruitment is increased. We used to have a TCM clinical trial which required the participants to take up to 12 TCM capsules daily for 6 months. We found the difficulty in recruitment increased significantly and compliance decreased.

Besides, many candidates were worried about whether the study herbal preparation could change their body nature, for example, let them become hot or cold. If they believed some ingredients in the formula could change their body nature, they might refuse to participate in the trial. To reduce their worries, we had a TCM practitioner to provide consultation to the participants during study period.

Some candidates wondered if certain foods should be avoided during taking the study herbal preparation. It would be inconvenient for participants if some herbal soups are to be avoided for several months. This was certainly a barrier for recruitment. Another barrier was the study duration. The efficacy of TCM was usually believed to be slow, which 
made the duration of the clinical trial longer. This keeps the subjects stay with the clinical trial a quite long time, and certainly increased the difficulty of recruitment and retention. Furthermore, long duration of trial might lead to delay of proper treatment, or worsening of the disease.

Randomized controlled trials of new treatment often include a control group receiving no treatment or placebo. However, as patients often assume that a new TCM treatment is likely to be effective, recruitment to trials with a placebo arm is more difficult than recruitment to those of active treatment ( Welton et al 1999). No one liked to be assigned to take placebo or go without treatment for a long-term period; they believed that they are likely to be exposed to a higher risk of disease deterioration compared with standard treatment. Therefore, greater reluctance from physicians and patients to participate in such designed study are expected (Roberts et al 2002; Hummer et al 2003). Some subjects were willing to participate in the trial but did not want to discontinue their concomitant complementary or alternative medicines, which they firmly believe would support their good health. So willingness to enter a randomized controlled trial of TCM would be lower when the trial included a placebo arm.

The barriers to recruitment also came from fear, distrust, or misunderstanding of the clinical trial process, unwilling to take herbal medication, unacceptable to some ingredient(s) of study TCM formula or they would not be treated like a "guinea pig". We conducted a randomized placebo controlled double blind clinical trial to investigate the efficacy of Coriolus Versicolor (Yunzhi) in breast cancer treatment. In this study, nearly $98 \%$ subjects were already taking other herbal drugs. They were reluctant to be randomized for fear of inclusion into the placebo group. Eventually, we had to compromise and change the inclusion criteria, allowed those taking herbal products to participate as long as they stick to use their herbal product without change during study period. From the point of view of clinical trial, this arrangement certainly caused bias; however these subjects were cancer patients, we had to balance ethics and research.

For some patients, the barriers would come from time constraints, travel problems, worry about overdoing the blood tests, too many visits scheduled, instead of an active medicine.

For clinicians, the main barriers come from too few patients being referred to the trial, too complicated outcome measurements, too little time to devote to recruitment activities, and too few patients having the disease or problem being tested. Insufficient recruitment was also due to Western doctors' unsatisfactory attitude to TCM study.
In Hong Kong, because of historical reasons, Western medicine is the official and mainstream healthcare provision. Western doctors trained in modern medicine have fewer opportunities to be trained in TCM. As they do not understand TCM well, they usually don't want their patients to take TCM before and during conventional treatment. This forms a barrier between modern medicine and TCM in Hong Kong. As a result, fewer candidates were referred to TCM clinical trials by Western doctors. For example, when we conducted cancer clinical trials using TCM as adjuvant treatment, we often had difficulties in patient recruitment because Western doctors did not encourage their patients to take TCM during conventional treatment.

There may be many clinical trials competing for these few patients who are willing to participate. Many physicians in Hong Kong usually do not encourage their patients to consider clinical trial participation, especially TCM clinical trials. These certainly increase the difficulties in subject recruitment for TCM clinical trials. Table 3 summarizes the difficulties in recruitment.

When insufficient recruitment occurred, we often consider the following steps which included reevaluating the required sample size; adding new sites to the trial; frequency of visits; extending the patient recruitment period, and modifying the patient inclusion/exclusion criteria. McDonald and colleagues (2006) reported that in about $10 \%$ of trials the inclusion criteria were changed or protocol amended to improve recruitment.

Attempts to enhance the recruitment the following strategies were tried: a) face-to-face contact with patients (eg, presentations at cancer support group); b) print contact at selected work sites (eg, flyers and posters distributed at community); c) notices in mass media (newspapers, journals, and broadcast media). In addition, the research team encouraged the participants to notify their friends and family members who might be eligible for enrollment. Posters and flyers were delivered to related medical clinics and nonprofit making and nongovernment organizations.

It was important to involve a registered TCM practitioner in a TCM clinical trial in order to keep the subjects clear from other herbal medicine/functional foods, with vague precise definition, and keep the subjects without withdrawal. The role of the TCM practitioner was to explain which herbal preparations or functional foods are to be avoided and provide consultation to the subjects while they were taking study medication. But no individualized prescription were given. This arrangement strengthened the relationship between subjects and investigators and the retention was much improved. 
Table 3 Reasons for recruitment barriers

\begin{tabular}{|c|c|c|}
\hline & Reasons for recruitment failure & $\begin{array}{l}\text { Occurred in our traditional Chinese medicine (TCM) } \\
\text { trials }\end{array}$ \\
\hline I & Unwilling to take herbal medication & $\begin{array}{l}\text { In a clinical trial of "Herbal Formulae KGL (抗感霊) } \\
\text { for Prevention and Treatment of Several Acute Respiratory } \\
\text { Syndrome", some health volunteers were unwilling to take } \\
\text { study herbal preparation because of the taste. }\end{array}$ \\
\hline 2 & Lack of interest in participating & $\begin{array}{l}\text { In Danggui Buxue Tang (棠歸補血湯) on symptomatic postmeno- } \\
\text { pausal trial and KGL (抗感靈) for prevention and treatment of } \\
\text { several acute respiratory syndrome trial, many candidates we } \\
\text { approached had no interest in clinical trials. }\end{array}$ \\
\hline 3 & $\begin{array}{l}\text { History of renal or liver diseases, or active gastrointestinal } \\
\text { diseases }\end{array}$ & $\begin{array}{l}\text { Some candidates who were eager to participate in TCM trial } \\
\text { were ineligible because of their renal and liver functions were } \\
\text { unsatisfactory to the inclusion criteria, eg, diabetic foot ulcer } \\
\text { trial, lung cancer trial and colorectal cancer trial. }\end{array}$ \\
\hline 4 & Out of the age range & $\begin{array}{l}\text { In asthma clinical trial, we had to exclude some candidates } \\
\text { because they were out of the age range. }\end{array}$ \\
\hline 5 & Participating in conflicting study & $\begin{array}{l}\text { Some candidates could not be recruited in Danggui Buxue } \\
\text { Tang (當歸補血湯) trial as they were participating in other trial of } \\
\text { hormone replacement therapy. }\end{array}$ \\
\hline 6 & Unwilling to commit to required time & $\begin{array}{l}\text { Some health volunteers or candidates whose problems were } \\
\text { not severe, were unwilling to follow the scheduled time, eg, } \\
\text { Danggui Buxue Tang (當歸補血晹) and KGL (抗感霝) trial. }\end{array}$ \\
\hline 7 & $\begin{array}{l}\text { Not willing to be randomized to nontreatment } \\
\text { or placebo group }\end{array}$ & $\begin{array}{l}\text { In the trial of Yunzhi and Danshen on immunological function } \\
\text { in breast cancer patients, nearly all subjects were unwilling } \\
\text { to take placebo. }\end{array}$ \\
\hline 8 & $\begin{array}{l}\text { Unacceptable to some ingredient(s) } \\
\text { of the TCM formula }\end{array}$ & $\begin{array}{l}\text { Due to intolerance of Danggui, some subjects refused to take } \\
\text { part in Danggui Buxue Tang (當歸補血湯) trial. }\end{array}$ \\
\hline 9 & $\begin{array}{l}\text { Refusal to traumatizing assessment, } \\
\text { eg, frequent blood taking }\end{array}$ & $\begin{array}{l}\text { Some health volunteers refused to participate in KGL (抗感霓) } \\
\text { trial as frequent blood taking was required. }\end{array}$ \\
\hline 10 & $\begin{array}{l}\text { Patient with significant medical problem, } \\
\text { eg, allergy }\end{array}$ & $\begin{array}{l}\text { Some candidates were excluded as they had history of } \\
\text { hypersensitivity to drugs (including herbal drugs), eg, asthma } \\
\text { and allergic rhinitis clinical trials. }\end{array}$ \\
\hline II & $\begin{array}{l}\text { Unable to comply with the protocol } \\
\text { requirements, eg, arranged visits, } \\
\text { appointed visit site, etc. }\end{array}$ & $\begin{array}{l}\text { Some candidates were unable to comply with the scheduled } \\
\text { visits because of working or trips, eg, in colorectal cancer trial } \\
\text { some subjects had to work and some wanted to travel. }\end{array}$ \\
\hline
\end{tabular}

Recruitment to trials might be increased if information given to potential participants was sufficient including the potential personal benefits and risks.

Frequent contact with subjects is essential for maintaining volunteer motivation to keep them stay in the study. We had a TCM practitioner involved in the trial to interpret the individual herb properties of the study herbal formulation and answer the questions raised by subjects during study period. This arrangement worked well in subject retention.

\section{Discussion: Possible solutions}

Traditional Chinese Medicine has a long history but its efficacy is not well-documented according to the requirements of evidence-based medicine (EBM). Proof of efficacy has to come from clinical trials (Ernst 2006). The design and reporting of clinical trials of TCM should follow the same guidelines as those for Western medicine (Ioannidis et al 2004). The diagnosis and treatment of TCM is based on overall analysis of the symptoms and signs. The treatments are frequently not standardized, but individualized and flexible, adjusting treatment as needed for the individual patient. In addition, there are wide variations and unrepeatable in practice. These are the common attitude of public. When we conduct a TCM clinical trial in compliance with randomized control trial principles, it is no doubt that we have a lot of barriers and difficulties in patient recruitment and retention apart from the clinical trial design. Based on our previous experience in TCM clinical trials we believe that the following issues may be helpful in patient recruitment.

\section{Proper evaluating inclusion criteria}

Inclusion criteria which are too strict may bar many subjects from entering the study. We used to exclude all subjects 
who were taking herbal preparation as dietary supplements. These strict inclusion criteria made the recruitment difficult because about $50 \%$ to $60 \%$ of the population in Hong Kong have consulted TCM practitioners (Wong et al 1995). According to a survey conducted by Critchley and colleagues (2005), in 259 surveyed adult Chinese patients admitted to a Hong Kong teaching hospital, 90\% used Chinese herbs on a regular daily basis in traditional soups and teas while $44 \%$ had consulted a TCM practitioner in the last twelve months prior to admission, but mainly for health promotion (59\%) and minor ailments (30\%). Only 25\% sought advice for their current illness and $13 \%$ were taking regular traditional Chinese medicines prior to admission. The ingredients were difficult to identify. The use of Chinese herbs in Chinese communities is very popular. If we exclude the subjects who take herbal preparations as dietary supplement or as medication, we would have great difficulties. To balance the recruitment accrual and result objectivity, we should encourage the eligible subject to stop taking other herbal preparations before and during study period. However, if the subject is not willing to quit, we had to advise the patient to just use the same herbal preparation at the same dosage throughout the study period. It would be useful for later data analysis if we had accurate information about the exact herbs being consumed.

Apart from the inclusion criteria, it was possible that recruitment effects were hampered by patients' perceived factors although these factors were not specific for TCM clinical trials as follows:

1. An exceedingly long clinical trial

2. Too many clinic visits

3. Too many tests conducted at each visit

4. Clinic visits that are inconvenient in terms of the number of hours required

5. Clinic visits that are inconvenient in terms of the time of day

6. Too great a chance of receiving a placebo instead of an active medicine.

\section{Improving the quality of interactions with patients}

All staff that interacted with patients during the recruitment process should be evaluated for the appropriateness and professionalism of their practice. This includes enthusiasm for the project, willingness to accept extra duties, empathy for the patient, and ability to discuss clearly the values and limitations of the trial. Experienced patients could be asked to share their experiences. We believe that the collaboration between research nurse and TCM practitioner in the process of patient recruitment and retention would significantly improve the efficiency and effect.

\section{Shifting placebo-controlled to active- controlled arrangements if possible}

A meta-analysis of dropout rate comparison in the active treatment and placebo treatment revealed that use of a placebo-controlled design had a major effect on the dropout rates (Kemmler et al 2005; McDonald et al 2006). The dropout rates may be higher in the placebo group than in the active group because of the lack of efficacy of placebo, which caused patients to leave the study prematurely. In recent years, ethical objections have been raised against the use of a placebo "treatment" once an efficacious standard treatment has been established. To replace the placebo-controlled arm with an active-controlled arm will be very helpful in patient retention if this change does not influence the outcome of the clinical trial.

\section{Interest and motivation}

Our patient population would have little incentive to participate in a placebo-controlled trial on TCM because they did not want to take the risk of being randomized to a placebo group. Subjects who believe the study herbal product may help them are likely to simply purchase the product from the market if it was commercially available. For incentive purposes we usually promise to provide the real medication free of change to participants who were randomized to receive the placebo at the end of the trial although this arrangement would not satisfy all.

In general, recruitment was difficult in clinical trials, and the refusal rate was high. A study reported (Mok et al 2007) that 322 patients were screened and offered consent, but only 120 agreed to participate. Seventy-nine patients $(39.2 \%)$ could not accept the placebo-controlled design, 45 patients $(22.3 \%)$ had already been taking Chinese herbal preparation before starting chemotherapy, and the rest either refused without a reason or were not interested. According a report (Wong et al 1995), 350 patients suitable for inclusion in the trial, $123(35 \%)$ refusal to take part and 51 (15\%) were excluded because they were unable to provide consent or relative assent.

Successful recruitment of participants is the key step to success in achieving the study aims. This paper described our reasoning, experiences, and the strategies we adopted to maximize recruitment during TCM clinical studies. Our experiences tell us that recruiting through the health services such as hospitals, clinics, general practitioners and 
nongovernmental organisations, is likely to be less successful than open advertising, as doctors in hospitals or clinics often have other priorities. Patient support groups may also be helpful. It is important that someone is assigned to telephone duty to deal with initial contact to avoid loss of potential volunteers. TCM clinical trials require a wide range of skills to successfully recruit subjects, in particular organization and oral communication skills. It is important to think ahead and to pre-empt volunteer queries. Frequent contact, including briefing and debriefing meetings is essential for maintaining volunteer motivation to stay in the study. The newspaper articles could attract attentions of potential participants within a short period and was faster in efficiency and less expensive. However since the article or interview was only placed a few days in a week, it is not possible to estimate how many people were exposed to this, so overall response rates cannot be determined.

\section{Conclusions}

Success in reaching target recruitment depended largely on being able to directly contact patients through media such as newspaper advertisements, television interviews, and physician referrals in hospitals. Suspicions against the possibility of being included in the placebo group, and unwillingness to stop taking other herbal supplements made recruitment more difficult, more time-consuming, and costly. In a Chinese community, open advertising in a local newspaper might be a particularly attractive option.

\section{Acknowledgments}

The authors would like to acknowledge the Ming Lai Foundation and The International Association of Lions Clubs District 303 - Hong Kong and Macau Tam Wah Ching Chinese Medicine Resource Centre for their never-ending support given to the Institute of Chinese Medicine, CUHK. The authors report no conflicts of interest.

\section{References}

Anderson DL. 2001. A Guide to Patient Recruitment: Today's Best Practices and Proven Strategies. Boston, MA: Centerwatch Inc.

Critchley LA, Chen DO, Lee A, et al. 2005. A survey of Chinese herbal medicine intake amongst preoperative patients in Hong Kong. Anaesth Intensive Care, 33:506-13.

Ernst E. 2006. Methodological aspects of traditional Chinese medicine (TCM). Ann Acad Med Singapore, 35:773-4.

Harris R, Dyson E. 2001. Methodological issues in nursing research Recruitment of frail older people to research: lessons learnt through experience. $J$ Adv Nurs, 36:643-51.

Hummer M, Holzmeister R, Kemmler G, et al. 2003. Attitudes of patients with schizophrenia toward placebo-controlled clinical trials. J Clin Psychiatry, 64:277-81.

Ioannidis JP, Evans SJ, Gøtzsche PC, et al. 2004. Better reporting of harms in randomized trials: an extension of the CONSORT statement. Ann Intern Med, 141:781-8.

Kemmler G, Hummer M, Widschwendter C, et al. 2005. Dropout rates in placebo-controlled and active-control clinical trials of antipsychotic drugs: A meta-analysis. Arch Gen Psychiatry, 62:1305-12.

Leung PC, Lau TW, Woo KS. 2008. Research on traditional Chinese medicine harmonizing two approaches. Oriental Pharm Experi Med, $8: 17-23$.

McDonald AM, Knight RC, Campbell MK, et al. 2006. What influences recruitment to randomized controlled trials? A review of trials funded by two UK funding agencies [online]. Accessed on April 7, 2007. URL: http://www.trialsjournal.com/content/7/1/9.

Mok TSK, Yeo W, Johnson PJ, et al. 2007. A double-blind placebo-controlled randomized study of Chinese herbal medicine as complementary therapy for reduction of chemotherapy-induced toxicity. Ann Oncol, 18:768-74.

Roberts LW, Warner TD, Brody JL, et al. 2002. Patient and psychiatrist ratings of hypothetical schizophrenia research protocols: assessment of harm potential and factors influencing participation decisions. AMJ Psychiatry, 159:573-84.

Ross S, Grant A, Counsell C, et al. 1999. Barriers to participation in randomized controlled trials: a systematic review. J Clin Epidemiol, 52:1143-56.

Spilker B, Cramer JA. 1992. Patient Recruitment in Clinical Trials. New York, NY: Raven Press.

Taylor-Davis S, Smicklas-Wright H, Davis AC, et al. 1998. Time and cost for recruiting older adults. $J$ Am Geriatr Soc, 46:753-7.

Welton AJ, Vickers MR, Cooper JA, et al. 1999. Is recruitment more difficult with a placebo arm in randomized controlled trials? A quasi-randomised interview based study. BMJ, 318:1114-7.

Wong TW, Wong SL, Donnan SP. 1995. Prevalence and determinants of the use of traditional Chinese medicine in Hong Kong. Asia Pac J Public Health, 8:167-70. 
